\title{
Electric-field-induced avalanches and glassiness of mobile ferroelastic twin domains in cryogenic $\mathrm{SrTiO}_{3}$
}

\author{
Blai Casals $\odot,{ }^{1}$ Sebastiaan van Dijken $\odot,{ }^{2}$ Gervasi Herranz $\odot,{ }^{3}$ and Ekhard K. H. Salje $\odot^{1}$ \\ ${ }^{1}$ Department of Earth Sciences, University of Cambridge, Cambridge CB2 3EQ, United Kingdom \\ ${ }^{2}$ NanoSpin, Department of Applied Physics, Aalto University School of Science, P. O. Box 15100, FI-00076 Aalto, Finland \\ ${ }^{3}$ Institut de Ciència de Materials de Barcelona (ICMAB-CSIC), Campus UAB, E-08193 Bellaterra, Catalonia, Spain
}

(Received 12 March 2019; revised manuscript received 26 June 2019; published 26 November 2019)

\begin{abstract}
Domain motion during ferroelectric switching has been recently suggested to follow scale-invariant avalanche dynamics. An interesting question concerns the dynamics of ferroelastic materials where the bulk material is nonpolar, while the polarity arises at domain walls only. We tackle this issue by investigating the dynamics of ferroelastic twins in $\mathrm{SrTiO}_{3}$ where the movement of domains is driven mainly by the anisotropic dielectric response at low temperatures. We find that the dynamics of the twin reconfiguration under electric field proceeds by jerks, where the energy distribution is power-law distributed, indicating avalanche dynamics. Avalanche exponents are sensitive to the complexity of the twin pattern structure, reflecting glassiness when twins are interwoven and forming junctions at the intersections between domain walls. This "glassy" behavior is attributed to the pinning originated by these self-generated defects during jamming between twins.
\end{abstract}

DOI: 10.1103/PhysRevResearch.1.032025

Patterns of ferroelastic twins can be changed by the application of electric fields in piezoelectric or electrostrictive materials [1,2]. Similar changes may also occur in pure nonpolar ferroelastics if, e.g., the dielectric parameters are anisotropic or the domain walls are polar. The latter situation is of particular interest, as it has been demonstrated that macroscopic polarity commonly exists in complex ferroelastic twin structures [3]. Indeed, field-induced changes of ferroelastic strains were reported for $\mathrm{CaTiO}_{3}$ [4], a definitely nonpolar material where twin walls carry polarity [5]. $\mathrm{SrTiO}_{3}$ is a particularly relevant case that comprises polar domain walls and a high dielectric anisotropy at low temperatures [6]. This material exhibits massive shifts of domain walls under applied electric fields, which go far beyond small bending effects as seen in friction experiments [7,8] and represent mesoscopic strain-driven domain movements [9-12].

These characteristics make of $\mathrm{SrTiO}_{3}$ an ideal candidate to analyze domain wall dynamics in a purely ferroelastic bulk material. Recently, it has been demonstrated that domain motion in some ferroelectrics follows scale-invariant avalanche dynamics [2]. An interesting question is to know if such behavior is mainly driven by the polarity of the bulk domains or, on the contrary, it is ruled by the properties of domain walls. In addition, the question arises whether other phenomena, such as the formation of self-generated pinning centers at domain wall junctions, may smooth or even suppress avalanche dyamics as predicted recently [13]. In this context, we should stress

Published by the American Physical Society under the terms of the Creative Commons Attribution 4.0 International license. Further distribution of this work must maintain attribution to the author(s) and the published article's title, journal citation, and DOI. that a glasslike behavior of polar domain walls in $\mathrm{SrTiO}_{3}$ has been observed for large relaxation times [14], which again raises the question about the character of the domain dynamics at much shorter timescales. We answer these questions by studying the dynamics of ferroelastic twin motion in $\mathrm{SrTiO}_{3}$. More specifically, we have exploited optical microscopy to show that the movements of domains are typical of avalanches with near-mean-field power-law distributions. Additionally, we show that there is a significant variation of the distribution depending on the complexity of the twin structure, so that simple patterns show pinning-depinning dynamics while complex patterns follow a more "glassy" behavior.

$\mathrm{SrTiO}_{3}$ undergoes a ferroelastic, nonferroelectric transition from a cubic to a tetragonal phase at around $105 \mathrm{~K}$. The transition follows mean-field behavior rather closely [15]. Ferroelastic twins are created during the transition and have two different orientations, namely, $\{a, c\}$ when the projection of domain walls (DWs) on the sample's plane $[001]_{c}$ are parallel to the crystallographic $[100]_{c}$ or $[010]_{c}$ axes and $\left\{a_{1}, a_{2}\right\}$ for walls parallel to $[110]_{c}$ or $[1 \overline{1} 0]_{c}$ using a pseudocubic unit cell. The electric field is applied perpendicular to the sample surface. Domain walls become polar below $80 \mathrm{~K}$ $[16,17]$ and their mobility under an electric field or external stress increases massively below $60 \mathrm{~K}[6,18,19]$. The twin reconfiguration under the electric field shows that $\{a, c\}$ twins retract and $\left\{a_{1}, a_{2}\right\}$ twins expand. The wall mobility is largely due to the dielectric anisotropy of $\mathrm{SrTiO}_{3}$ at low temperatures as discussed in detail in Ref. [6].

Experimentally, we image large areas of (001)- $\mathrm{SrTiO}_{3}$ optically using a CCD camera with domain movements followed with a time resolution of 12 frames/s. The electric field (rate of $16 \mathrm{~V} / \mathrm{mm} \mathrm{s}$ ) was applied perpendicular to the sample surface through two thin $\mathrm{Cr}$ (5-nm) contacts deposited by chemical vapor deposition (CVD) evaporation. 
(a)

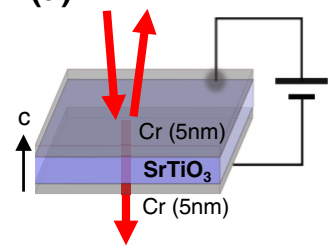

(b) Reflection

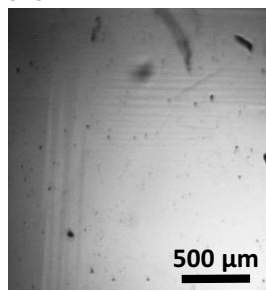

(d) Case A: Simple twin pattern

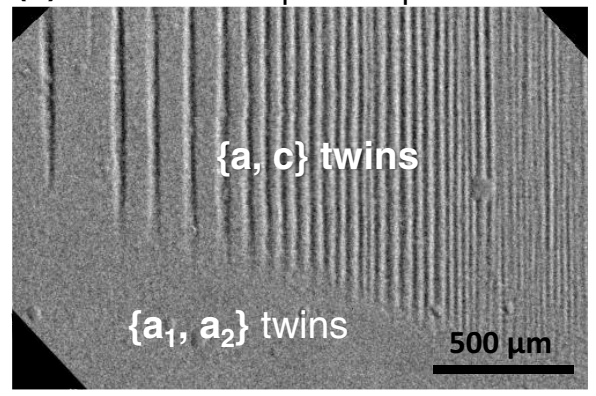

$\{a, c\}$ twins
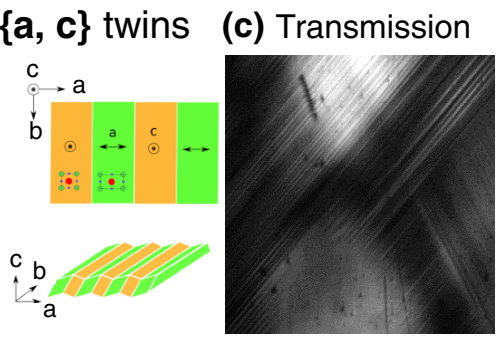

$\left\{a_{1}, a_{2}\right\}$ twins

$c$

$\leftrightarrow$ a

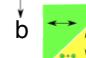

$\because \hat{A}^{a_{1}}$

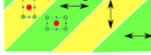

b

a (e) Case B: Complex twin pattern

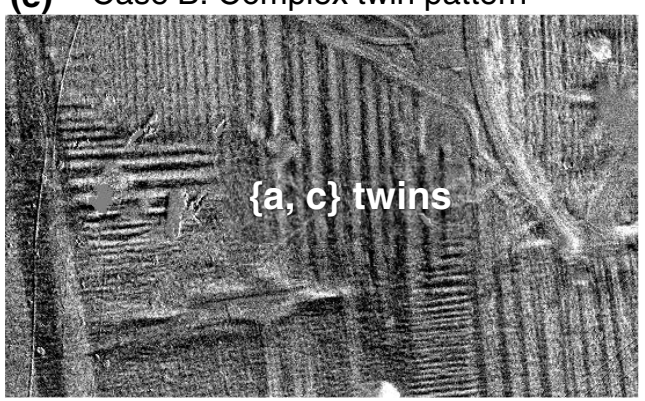

FIG. 1. (a) Experimental configuration. The electric field is applied perpendicular to the sample surface using two Cr electrodes. The red arrows indicate the light pathway in reflection and transmission. (b) Reflection image showing topographic contrast from $\{a, c\}$ twins. (c) Transmission image showing birefringence contrast from $\left\{a_{1}, a_{2}\right\}$ twins. (d) Case A: Sample area where the twin pattern is formed by two big areas with simple twin patterns of $\{a, c\}$ and $\left\{a_{1}, a_{2}\right\}$ (the latter pattern is not visible in this reflection measurement). (e) Case B: Sample area where the twin pattern is formed by an intricate structure of $\{a, c\}$ twins. The reflection images in (d) and (e) were recorded at $6 \mathrm{~K}$.

Both electrodes were thin enough to allow the observation of twins in optical transmission. The experimental arrangement is shown in Fig. 1(a). Due to the properties of the twin orientations, optical microscopy distinguishes between $\{a, c\}$ and $\left\{a_{1}, a_{2}\right\}$ twins. The $\{a, c\}$ twins create a corrugation in the sample surface that can be observed topographically via surface reflection [Fig. 1(b)]. $\left\{a_{1}, a_{2}\right\}$ twins do not exhibit such surface topography and therefore cannot be seen by light reflection. By working in a transmission mode with polarized light, $\left\{a_{1}, a_{2}\right\}$ twins are imaged by changes in birefringence [Fig. 1(c)], whereas $\{a, c\}$ twins are almost undistinguishable by birefringence. Figures 1(b) and 1(c) show the reflection and the birefringence images, respectively, taken at the same $\mathrm{SrTiO}_{3}$ sample. In Fig. 1(b) there is an area without $\{a, c\}$ twinning contrast corresponding to a flat area with $\left\{a_{1}, a_{2}\right\}$ twins. Similarly, an area without $\left\{a_{1}, a_{2}\right\}$ twins but only $\{a, c\}$ twins are shown in Fig. 1(c). The images reveal twin patterns of different complexity. Case A represents the simplest structure where stripe domains form patterns with twinning along the same direction. Figure 1(d) shows $\{a, c\}$ twinning imaged in reflection. Conversely, more complex patterns are observed in case $\mathrm{B}$, where a complex twin pattern is formed by an intricate superposition of $\{a, c\}$ twins in two orthogonal directions [Fig. 1(e)]. In the following we analyze each case separately.

We analyzed the twin mobility under electric field as a function of temperature. By way of illustration, in Fig. 2 we show the topography changes obtained by subtracting the reflection images recorded at $400 \mathrm{~V} / \mathrm{mm}$ from that at $0 \mathrm{~V} / \mathrm{mm}$. These images hence show the difference between twin patterns and relate to the movement of domains. At 6 and $20 \mathrm{~K}$ large field-induced differences are found while just a few changes occur at $40 \mathrm{~K}$ and no domain rearrangements are observed at temperatures above $60 \mathrm{~K}$. We have quantified the mobility by the percentage of change between 0 and $400 \mathrm{~V} / \mathrm{mm}$ of the mean intensity of the subtraction images. As shown in Fig. 2(b), the mobility rapidly decays above $40 \mathrm{~K}$. We then studied the twin motion under electric field at low temperatures in the high mobility regime.

First, we focused on the optical reflection of $\{a, c\}$ twins (case A). Figure 3(a) shows the subtracted images taken at increasing voltages from 0 to $400 \mathrm{~V} / \mathrm{mm}$ minus the image at $0 \mathrm{~V} / \mathrm{mm}$. Hence, the images show topographical changes, so

(a) Topography changes
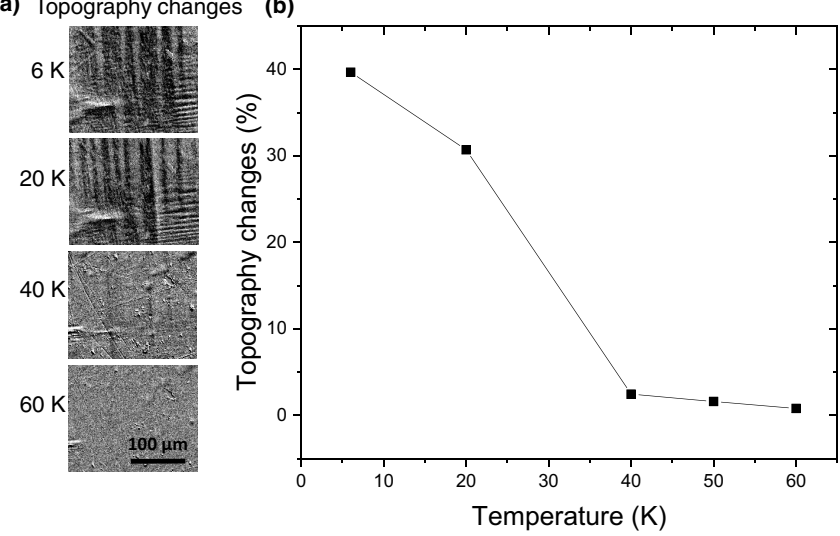

FIG. 2. (a) Topographical changes obtained by subtracting reflection images recorded at $400 \mathrm{~V} / \mathrm{mm}$ from $0 \mathrm{~V} / \mathrm{mm}$ (case B twin pattern). The field of view contains an intricate pattern of $\{a, c\}$ twins. Strong contrast in the subtracted images (a) at 6 and $20 \mathrm{~K}$ indicates strong twin motion. Few changes occur at $40 \mathrm{~K}$ and none at $60 \mathrm{~K}$. (b) Topography changes defined as the percentage of the mean intensity of subtracted images in (a). 
(a) $\mathrm{I}(0 \mathrm{~V} / \mathrm{mm})-1(0 \mathrm{~V} / \mathrm{mm})$

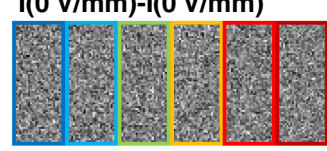

$\mathrm{I}(100 \mathrm{~V} / \mathrm{mm})-\mathrm{I}(0 \mathrm{~V} / \mathrm{mm})$

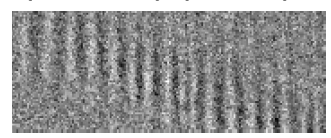

$\mathrm{I}(200 \mathrm{~V} / \mathrm{mm})-\mathrm{I}(0 \mathrm{~V} / \mathrm{mm})$

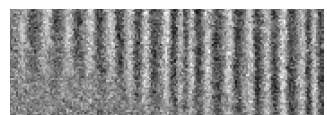

$\mathrm{I}(400 \mathrm{~V} / \mathrm{mm})-\mathrm{I}(0 \mathrm{~V} / \mathrm{mm})$

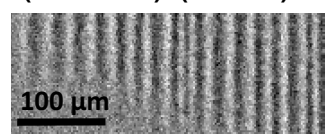

(b)

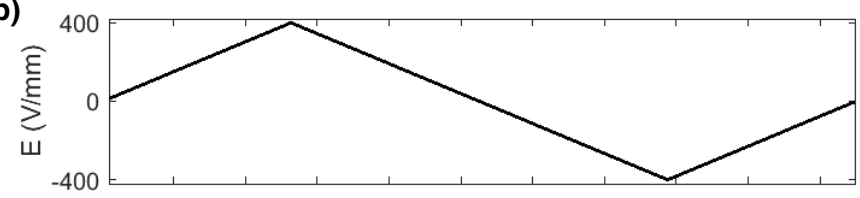

(c)
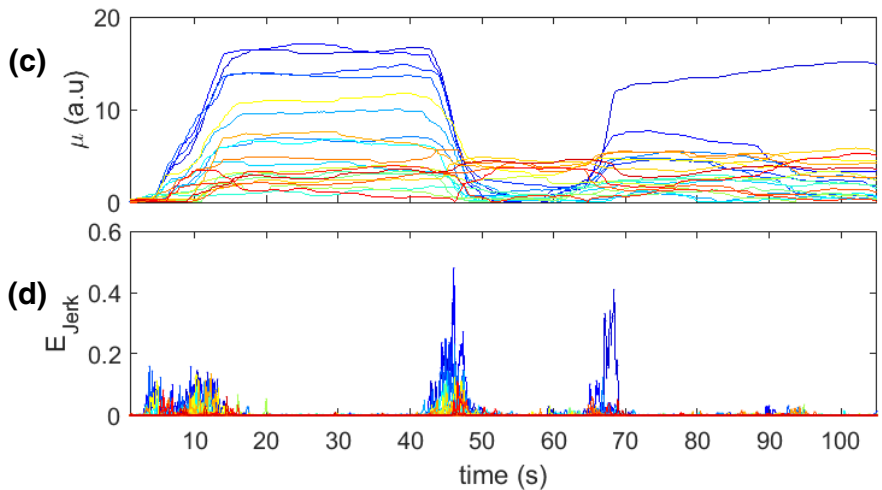

(e)

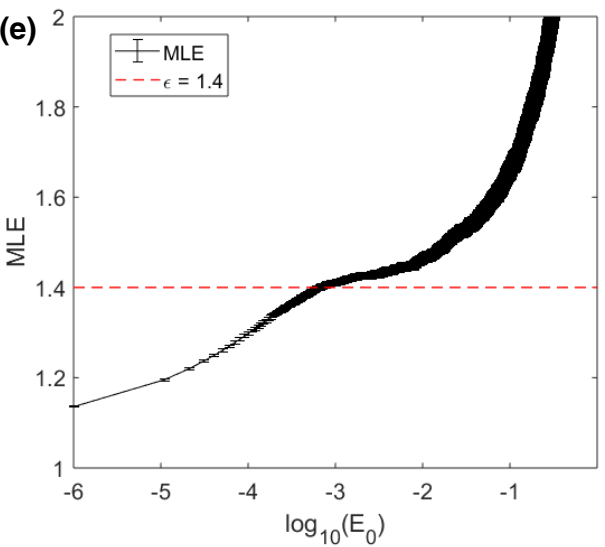

(f)

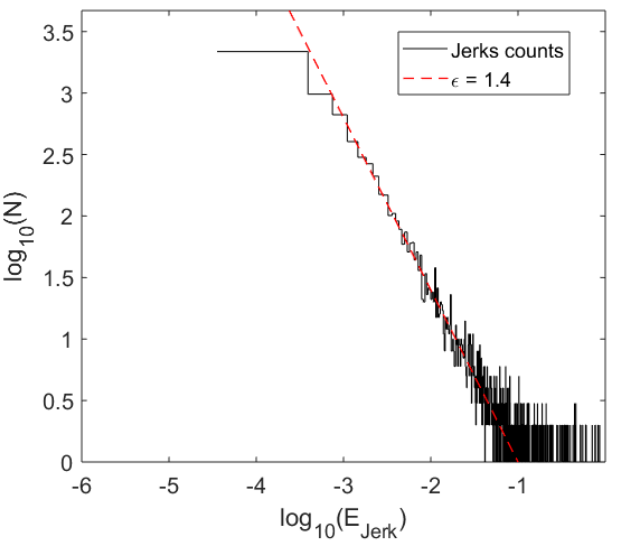

FIG. 3. (a) Topographical changes of the $\{a, c\}$ twins under electric field taken by subtracting images at increasing voltages from 0 to $400 \mathrm{~V} / \mathrm{mm}$ from the image at $0 \mathrm{~V} / \mathrm{mm}$. (b) Applied voltage as a function of time. (c) The mean value $(\mu)$ is computed in all the regions schematically shown in (a) as a function of time. The color code indicates the regions in (a) from blue to red. (d) The jerk spectra ( $\left.E_{\text {jerks }}\right)$ of moving twins. (e) Maximum likelihood estimated exponent (MLE) for the $\{a, c\}$ twins as a function of the varying energy cutoff ( $\left.E_{0}\right)$ in black error bars. The dashed red line indicates the best chosen exponent of 1.4. (f) Log-log plot for the jerk spectra distribution (black) and the best fit for the largest energy range of a slope of -1.4 (red dashed).

that $\{a, c\}$ twin domains retract with increasing field [6]. At $100 \mathrm{~V} / \mathrm{mm}$ they have retracted from half of the area, at $200 \mathrm{~V} / \mathrm{mm}$ all twins have disappeared, and no additional changes occur between 200 and $400 \mathrm{~V} / \mathrm{mm}$. We cycled the electric field between 400 and $-400 \mathrm{~V} / \mathrm{mm}$ in a triangular fashion [Fig. 3(b)]. In order to study the motion of the twins we divided the image into regions parallel to the twins with a width similar to each twin [see the colored rectangles in Fig. 3(a)]. For each region we computed the mean value $(\mu)$ as an indicator of topographic changes as a function of the applied voltage. A similar procedure is commonly used in speckle spectra analysis [20-22]. These changes are shown in Fig. 3(c) where it becomes obvious that twins move through jumps rather than by smooth propagation $[9,13]$. These steps define "jerks" where each jerk corresponds to an avalanche of domain motions [9,11]. We established a data set of all jerk amplitudes by computing the first derivate of the mean value $\left(A_{\text {jerks }}=d \mu / d t\right)$ and its corresponding energies [Fig. 3(d)] as the square of the amplitudes $\left(E_{\text {jerks }}=A_{\text {jerks }}^{2}\right)$. Typically, Fig. 3(d) shows few jerks with large energies and many small energy jerks. Quantitatively, the jerk motion is power-law distributed with the probability distribution per energy interval $\operatorname{PDF}(E)=E_{0} E^{-\varepsilon}$, where $\varepsilon$ is the avalanche energy exponent which, in mean-field theory, is predicted to be $\varepsilon=4 / 3$ [23]. In order to estimate $\varepsilon$ from the experimental data and verify whether it is in agreement with scale-invariant mean-field models, we use the maximum likelihood method (ML) expressed in Eq. (1),

$$
\hat{\varepsilon}=1+N_{E \geqslant E_{0}}\left[\sum_{i=1}^{N_{E \geqslant E_{0}}} \ln \left(\frac{E_{i}}{E_{0}}\right)\right],
$$

where $\hat{\varepsilon}$ is the estimated energy exponent (MLE), $E_{0}$ is a varying energy cutoff, and $N_{E>E_{0}}$ the number of jerks with equal or higher energy than $E_{0}$.

Figure 3(e) shows the MLE for jerk spectra composed by all jerks in reflection. An onset of a plateau can be seen at $\varepsilon=1.4$, which is near the mean-field value 1.33. An alternative way to estimate the power-law exponent is to plot 

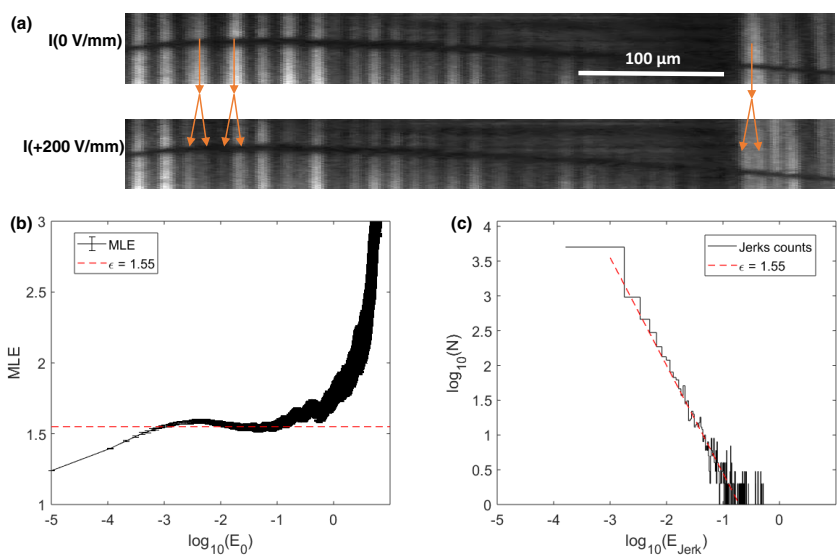

FIG. 4. (a) Birefringence image at 0 and at $200 \mathrm{~V} / \mathrm{mm}$. The arrows indicate the $\left\{a_{1}, a_{2}\right\}$ twins which double their period at $200 \mathrm{~V} / \mathrm{mm}$. (b) MLE analysis (black error bars) showing a plateau expanding along two decades indicated by the red dashed line. (c) Log-log plot for the jerk spectra distribution (black line). The best fit at the same energy range as the MLE plateau corresponds to a slope of -1.55 (red dashed line).

the distribution of the amount of jerks of a certain energy as a function of energy in a log-log plot. The exponent is then obtained by linear regression. The experimental distribution of jerks is shown in Fig. 3(f) where the slope corresponds to $\varepsilon=1.4$. The power law indicates scale-invariant with cutoff effects at small and large energies [24]. At low energies, the amount of the experimental noise is comparable with the jerk energies and causes a deflection in the log-log plot. At high energies, only a few jerks were measured and therefore the effect of low sample statistics becomes relevant. In summary, we showed that field-induced $\{a, c\}$ twin motion progresses by avalanches with an energy exponent close to the mean-field value.

We now focus on the dynamics of $\left\{a_{1}, a_{2}\right\}$ twins in the simple pattern configuration (case A). These twins expand under the applied electric field and occupy the empty areas left by the $\{a, c\}$ twins. Figure 4(a) shows the birefringence images at 0 and $200 \mathrm{~V} / \mathrm{mm}$ that reveal the presence of the $\left\{a_{1}, a_{2}\right\}$ twins. A little scratch in the Cr electrode shows that both images correspond to the same area. As indicated by the arrows in Fig. 4(a), the main effect is period doubling of the sequence of thin walls [25-31]. Applying the same procedure as before, we tracked the birefringence as a function of time while cycling the electric field and constructed the jerk spectra (see details in the Supplemental Material [32]). Computing the MLE, a plateau that expands along two decades was found with $\varepsilon=1.55$ [Fig. 4(b)]. A linear behavior is observed for log-log binning [Fig. 4(c)] with the same energy exponent. The dynamical motions of $\{a, c\}$ and $\left\{a_{1}, a_{2}\right\}$ twins under electric field follow, thus, the scale-invariant power-law statistics characteristic of the avalanche dynamics. The exponents are slightly different (1.4 and 1.55$)$, respectively.

In case $\mathrm{B}$, we observed a few jerks from the domain movement under electric field. The twins move with the field in a glassier, or viscous, fashion and are less jerky than for the simple domain pattern. In order to have a noise reference, we analyzed the twin motion at low temperatures (6 and $20 \mathrm{~K}$ )
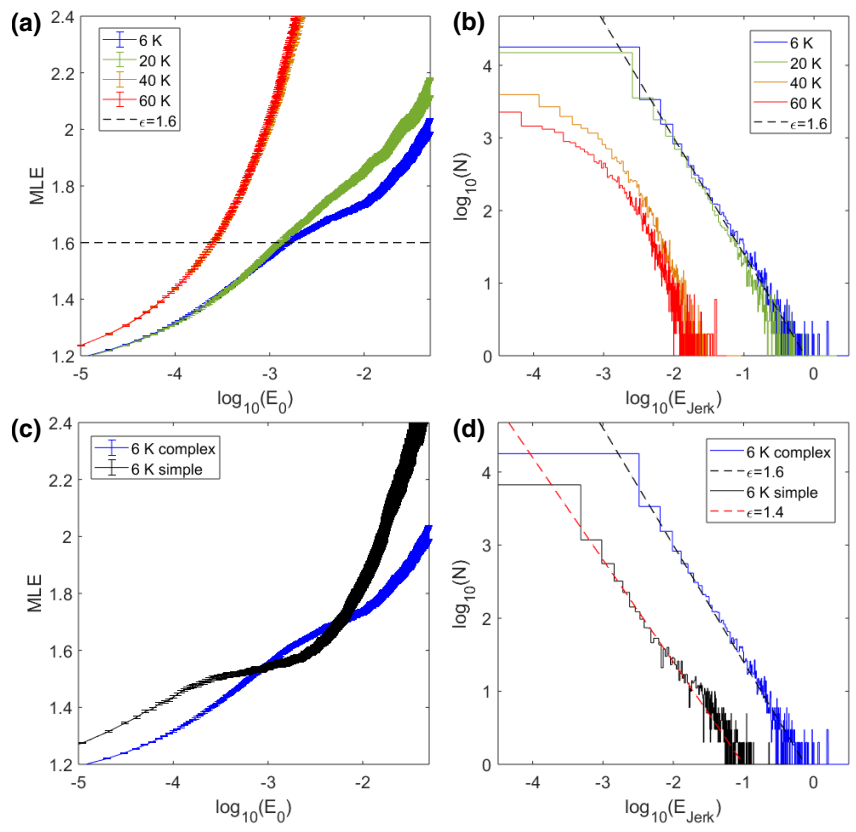

FIG. 5. Jerk analysis on the complex twin pattern (case B) at 6, 20, 40, and $50 \mathrm{~K}$. (a) MLE analysis of the jerks where a deflection is observed near $\varepsilon=1.6$ for temperatures below $40 \mathrm{~K}$. (b) Log-log binning plots for all temperatures. A power law with $\varepsilon=1.6$ (dashed red line) is fitted at $6 \mathrm{~K}$. (c), (d) Comparison of the MLE and log-log binning plots obtained for the complex (blue data) and simple (black data) twin patterns.

and above $40 \mathrm{~K}$ where the twins are not mobile under the applied electric field (Fig. 2). Applying the same analysis as before (see details in the Supplemental Material [32]), the ML does show a deflection close to $\varepsilon=1.6$ [Fig. 5(a) at 6 and $20 \mathrm{~K}]$. This deflection is attributed to an exponentially damped power law [33]. For temperatures above $40 \mathrm{~K}$ the ML does not show any plateau or deflection with behavior compatible with noise. Similarly, the log-log plot [Fig. 5(b)] shows linear behavior fitted with a power law with $\varepsilon=1.6 \pm$ 0.05 at 6 and at $20 \mathrm{~K}$. In the nonmobile region (above $40 \mathrm{~K}$ ), the log-log plot shows nonlinear behavior shifted towards lower energies, which corresponds to noise since no jerks are seen in the twin motion. Both ML and log-log methods estimate an energy exponent around $\varepsilon=1.6$ with very high damping [33]. We attribute this damping to intrinsic effective pinning generated by the intersections and junctions of the twin $[13,34]$.

Both exponents, namely, 1.4 and 1.6 for cases A and B, respectively, are remarkably close to the mean-field values, which, as discussed next, have been observed before. More specifically, a value of $4 / 3$ relates to the mean-field prediction of fast processes, where the switching of domains is an instantaneous process [23]. No ferroelectric switching exponents of 1.4 were reported before while this value is typical for local collapse such as around holes in porous materials. Several previous papers confirm collapse data with this exponent [35-38] by acoustic emission studies. In contrast, domain switching often requires the relaxation of the matrix between the domain movement. This scenario is captured by the "field-integrated" mean-field exponent 1.6 [23]. This value was previously 
observed for switching in $\mathrm{BaTiO}_{3}$ [2], lead zirconate titanate (PZT) [39], and in stress-induced switching in ferroelastics $[11,12]$. The most important example is probably $\mathrm{LaAlO}_{3}$ $[9,10]$ where the switching exponents were measured under simultaneous observation of the needle progression. Most importantly, the domain movements in scenario B also display large damping, which is absent in scenario A. This shows that not only do significant relaxations occur when domain walls intersect, but that a large energy transfer occurs during the switching process. Such large damped avalanches are the hallmark of glassy materials.

In summary, we have analyzed different representative cases presenting different complexities of ferroelastic twin patterns. The relevant energy exponents vary between 1.4 and 1.6. The reproducibility of the energy exponents is around 0.05 . For relatively simple patterns the twin reconfiguration under electric field follows a jerky motion obeying a powerlaw distribution with $\varepsilon=1.4$. Similar avalanche behavior occurs for both retracting $\{a, c\}$ and expanding $\left\{a_{1}, a_{2}\right\}$ twins that double their period in a comb configuration. The period doubling shows a larger exponent. Currently we are undertaking computer simulations to identify the mechanism that leads to the increase of the energy exponent. On the other hand, in the case of more complex patterns, the twin reconfiguration under electric field produces jerks obeying a power law with a larger energy exponent $(\varepsilon=1.6)$ compared to the simple twin pattern but strongly increased damping. We attributed this difference to the pinning by self-generated defects (junctions) and thus we report here an experimental example of previous predictions [13]. Our results are in close analogy to similar studies on ferroelectric $\mathrm{BaTiO}_{3}$ [2], where the avalanches are driven by the ferroelectric switching due to the bulk polarity, absent in $\mathrm{SrTiO}_{3}$, where the main driving force is the dielectric anisotropy at low temperatures [6]. As a conclusion, the $\mathrm{SrTiO}_{3}$ twin reconfiguration under electric field at temperatures below $40 \mathrm{~K}$ proceeds via jerks (avalanches) and its glassy behavior strongly depends on the complexity of the twin pattern.

We acknowledge financial support from EPSRC (Grant No. EP/P024904/1) and support from the Spanish Ministerio de Ciencia, Innovación y Universidades through Grant No. MAT2017-85232-R (AEI/FEDER, EU), Severo Ochoa SEV-2015-0496, the Generalitat de Catalunya through Grant No. 2017301 SGR1377, and the Academy of Finland (Grant No. 316857$)$.
[1] O. Aktas, J. R. Duclere, S. Quignon, G. Trolliard, and E. K. H. Salje, Polarity of modulated $\mathrm{Na}_{0.5} \mathrm{Bi}_{0.5} \mathrm{TiO}_{3}$ and its slow structural relaxation, Appl. Phys. Lett. 113, 032901 (2018).

[2] E. K. H. Salje, D. Xue, X. Ding, K. A. Dahmen, and J. F. Scott, Ferroelectric switching and scale invariant avalanches in $\mathrm{BaTiO}_{3}$, Phys. Rev. Mater. 3, 014415 (2019).

[3] E. K. H. Salje, S. Z. Li, M. Stengel, P. Gumbsch, and X. D. Ding, Flexoelectricity and the polarity of complex ferroelastic twin patterns, Phys. Rev. B 94, 024114 (2016).

[4] E. K. H. Salje, O. Aktas, and X. Ding, Functional topologies in (multi-) ferroics: The ferroelastic template, in Topological Structures in Ferroic Materials, edited by J. Seidel, Springer Series in Materials Science Vol. 228 (Springer, Berlin, 2016), p. 83.

[5] E. K. H. Salje, Functional twin boundaries: Steps towards domain boundary engineering, in Mesoscopic Phenomena in Multifunctional Materials, edited by A. Saxena and A. Planes, Springer Series in Materials Science Vol. 198 (Springer, Berlin, 2014), p. 201.

[6] B. Casals, A. Schiaffino, A. Casiraghi, S. J. Hamalainen, D. L. Gonzalez, S. van Dijken, M. Stengel, and G. Herranz, LowTemperature Dielectric Anisotropy Driven by an Antiferroelectric Mode in $\mathrm{SrTiO}_{3}$, Phys. Rev. Lett. 120, 217601 (2018).

[7] S. Kustov, E. Cesari, I. Liubimova, V. Nikolaev, and E. K. H. Salje, Twinning in Ni-Fe-Ga-Co shape memory alloy: Temperature scaling beyond the Seeger model, Scr. Mater. 134, 24 (2017).

[8] S. Kustov, D. Salas, E. Cesari, R. Santamarta, D. Mari, and J. Van Humbeeck, Structural anelasticity, elasticity and broken ergodicity in Ni-Ti shape memory alloys, Acta. Mater. 73, 275 (2014).

[9] R. J. Harrison and E. K. H. Salje, The noise of the needle: Avalanches of a single progressing needle domain in $\mathrm{LaAlO}_{3}$, Appl. Phys. Lett. 97, 021907 (2010).
[10] R. J. Harrison and E. K. H. Salje, Ferroic switching, avalanches, and the Larkin length: Needle domains in $\mathrm{LaAlO}_{3}$, Appl. Phys. Lett. 99, 151915 (2011).

[11] S. Puchberger, V. Soprunyuk, W. Schranz, A. Troster, K. Roleder, A. Majchrowski, M. A. Carpenter, and E. K. H. Salje, The noise of many needles: Jerky domain wall propagation in $\mathrm{PbZrO}_{3}$ and $\mathrm{LaAlO}_{3}$, APL Mater. 5, 046102 (2017).

[12] E. K. H. Salje, J. Koppensteiner, M. Reinecker, W. Schranz, and A. Planes, Jerky elasticity: Avalanches and the martensitic transition in $\mathrm{Cu}_{74.08} \mathrm{Al}_{23.13} \mathrm{Be}_{2.79}$ shape-memory alloy, Appl. Phys. Lett. 95, 231908 (2009).

[13] E. K. H. Salje, X. Ding, Z. Zhao, T. Lookman, and A. Saxena, Thermally activated avalanches: Jamming and the progression of needle domains, Phys. Rev. B 83, 104109 (2011).

[14] D. Pesquera, M. A. Carpenter, and E. K. H. Salje, Glasslike Dynamics of Polar Domain Walls in Cryogenic $\mathrm{SrTiO}_{3}$, Phys. Rev. Lett. 121, 235701 (2018).

[15] E. K. H. Salje, M. C. Gallardo, J. Jimenez, F. J. Romero, and J. del Cerro, The cubic-tetragonal phase transition in strontium titanate: Excess specific heat measurements and evidence for a near-tricritical, mean field type transition mechanism, J. Phys.: Condens. Matter 10, 5535 (1998).

[16] E. K. H. Salje, O. Aktas, M. A. Carpenter, V. V. Laguta, and J. F. Scott, Domains within Domains and Walls within Walls: Evidence for Polar Domains in Cryogenic $\mathrm{SrTiO}_{3}$, Phys. Rev. Lett. 111, 247603 (2013).

[17] J. F. Scott, E. K. H. Salje, and M. A. Carpenter, Domain Wall Damping and Elastic Softening in $\mathrm{SrTiO}_{3}$ : Evidence for Polar Twin Walls, Phys. Rev. Lett. 109, 187601 (2012).

[18] H. J. H. Ma, S. Scharinger, S. W. Zeng, D. Kohlberger, M. Lange, A. Stohr, X. R. Wang, T. Venkatesan, R. Kleiner, J. F. Scott, J. M. D. Coey, D. K. Ariando, and Ariando, Local Electrical Imaging of Tetragonal Domains and Field-Induced 
Ferroelectric Twin Walls in Conducting $\mathrm{SrTiO}_{3}$, Phys. Rev. Lett. 116, 257601 (2016).

[19] M. Honig, J. A. Sulpizio, J. Drori, A. Joshua, E. Zeldov, and S. Ilani, Local electrostatic imaging of striped domain order in $\mathrm{LaAlO}_{3} / \mathrm{SrTiO}_{3}$, Nat. Mater. 12, 1112 (2013).

[20] R. Bandyopadhyay, A. S. Gittings, S. S. Suh, P. K. Dixon, and D. J. Durian, Speckle-visibility spectroscopy: A tool to study time-varying dynamics, Rev. Sci. Instrum. 76, 093110 (2005).

[21] H. Yang, R. Li, P. Kong, Q. C. Sun, M. J. Biggs, and V. Zivkovic, Avalanche dynamics of granular materials under the slumping regime in a rotating drum as revealed by speckle visibility spectroscopy, Phys. Rev. E 91, 042206 (2015).

[22] H. Katsuragi and D. J. Durian, Unified force law for granular impact cratering, Nat. Phys. 3, 420 (2007).

[23] E. K. H. Salje and K. A. Dahmen, Crackling noise in disordered materials, Annu. Rev. Condens. Matter Phys. 5, 233 (2014).

[24] E. K. H. Salje, H. L. Liu, L. S. Jin, D. Y. Jiang, Y. Xiao, and $\mathrm{X}$. Jiang, Intermittent flow under constant forcing: Acoustic emission from creep avalanches, Appl. Phys. Lett. 112, 054101 (2018).

[25] E. K. H. Salje, On the kinetics of partially conserved order parameters - a possible mechanism for pattern-formation, J. Phys.: Condens. Matter 5, 4775 (1993).

[26] Q. M. Wang and X. H. Zhao, A three-dimensional phase diagram of growth-induced surface instabilities, Sci. Rep. 5, 8887 (2015).

[27] I. Oprea and G. Dangelmayr, A period doubling route to spatiotemporal chaos in a system of Ginzburg-Landau equations for nematic electroconvection, Discrete Continuous Dyn. Syst. B 24, 273 (2019).

[28] S. Villain-Guillot, 1D Cahn-Hilliard equation: Ostwald ripening and modulated phase systems, Int. J. Bifurcation Chaos Appl. Sci. Eng. 19, 3541 (2009).

[29] A. I. Olemskoi and V. F. Klepikov, The theory of spatiotemporal pattern in nonequilibrium systems, Phys. Rep. 338, 571 (2000).
[30] E. K. H. Salje, M. T. Dove, I. Tsatskis, K. Locherer, and J. Chrosch, Fluctuations and some strain related interaction mechanisms in structural phase transitions, Phase Transitions 67, 539 (1999).

[31] I. Tsatskis, E. K. H. Salje, and V. Heine, Pattern-formation during phase-transitions-Kinetics of partially conserved order parameters and the role of gradient energies, J. Phys.: Condens. Matter 6, 11027 (1994).

[32] See Supplemental Material at http://link.aps.org/supplemental/ 10.1103/PhysRevResearch.1.032025 for the as-measured data for Figs. 4 and 5.

[33] E. K. H. Salje, A. Planes, and E. Vives, Analysis of crackling noise using the maximum-likelihood method: Power-law mixing and exponential damping, Phys. Rev. E 96, 042122 (2017).

[34] E. K. H. Salje, X. F. Wang, X. D. Ding, and J. F. Scott, Ultrafast switching in avalanche-driven ferroelectrics by supersonic kink movements, Adv. Funct. Mater. 27, 1700367 (2017).

[35] G. F. Nataf, P. O. Castillo-Villa, J. Baro, X. Illa, E. Vives, A. Planes, and E. K. H. Salje, Avalanches in compressed porous $\mathrm{SiO}_{2}$-based materials, Phys. Rev. E 90, 022405 (2014).

[36] J. Baro, A. Corral, X. Illa, A. Planes, E. K. H. Salje, W. Schranz, D. E. Soto-Parra, and E. Vives, Statistical Similarity between the Compression of a Porous Material and Earthquakes, Phys. Rev. Lett. 110, 088702 (2013).

[37] Y. Chen, X. D. Ding, D. Q. Fang, J. Sun, and E. K. H. Salje, Acoustic emission from porous collapse and moving dislocations in granular $\mathrm{Mg}-\mathrm{Ho}$ alloys under compression and tension, Sci. Rep. 9, 1330 (2019).

[38] X. Jiang, H. L. Liu, I. G. Main, and E. K. H. Salje, Predicting mining collapse: Superjerks and the appearance of recordbreaking events in coal as collapse precursors, Phys. Rev. E 96, 023004 (2017).

[39] C. D. Tan, C. Flannigan, J. Gardner, F. D. Morrison, E. K. H. Salje, and J. F. Scott, Electrical studies of Barkhausen switching noise in ferroelectric PZT: Critical exponents and temperature dependence, Phys. Rev. Mater. 3, 034402 (2019). 